\title{
Visible light induced electron transfer behavior of a CeO2-loaded HfO2/carbon cluster nanocomposite material
}

\begin{abstract}
The microwave-irradiated calcination of $\mathrm{HfOCl} / \mathrm{starch}$ complex I under an air atmosphere produced the $\mathrm{HfO} 2 /$ carbon cluster composite material which is denoted as Ic. The obtained composite material could decompose methylene blue under the irradiation of light $(\lambda>460$ $\mathrm{nm})$. The surface of Ic was loaded with $\mathrm{CeO} 2$ particles to obtain $\mathrm{CeO} 2$-loaded composite material, which can decompose the aqueous silver nitrate solution and produce $\mathrm{O} 2$ and $\mathrm{Ag}$ in the ratio of 1:4.2. Water photo-decomposition experiment was also carried out using Ptmodified composite materials.
\end{abstract}

Keyword: Semiconductors; Polymers; Nanostructures; Inorganic compounds; Electronic structure 